\title{
Locking compression plate distal ulna hook plate fixation versus intramedullary screw fixation for displaced avulsion fifth Metatarsal Base fractures: a comparative retrospective cohort study
}

\author{
Lin Xie ${ }^{\dagger}$, Xin Guo ${ }^{\dagger}$, Shu-Jun Zhang and Zhen-Hua Fang ${ }^{*}$
}

\begin{abstract}
Background: Intramedullary screw (IMS) fixation was wildly used in fifth metatarsal base fractures (FMBFs) and the results were satisfactory. However, in the comminuted osteoporosis or small displaced avulsion FMBFs, anatomical reduction and stable fixation could not be achieved with IMS. The Locking Compression Plate (LCP) distal ulna hook plate fixation was a novel alternative fixation method. The aim of this retrospective cohort study was to determine if LCP distal ulna hook plate fixation resulted in improved outcomes compared to the traditional IMS fixation in displaced avulsion FMBFs.

Methods: Of 43 patients with displaced avulsion FMBFs, 18 patients were treated with LCP distal ulna hook plate fixation and 25 were treated with IMS fixation. The patients were evaluated clinically and radiographically and followed up to 12 months. The surgery time, time for hospital stay, time for weight-bearing, time for bony union, time for return to daily life, pain relief, functional outcome and complications after treatment with LCP distal ulna hook plate fixation or IMS fixation were compared. The functional outcome was assessed by the AOFAS (American Orthopedic Foot and Ankle Society) mid-foot score at 3, 6, 9, and 12 months after surgery. Meanwhile, pain scores were obtained at 3, 6, 9, and 12 months after surgery.

Results: The two cohorts had similar baseline characteristics. Surgery time was less in LCP distal ulna hook plate fixation cohort compare to IMS fixation cohort $(p<0.0001)$. Time for partial weight-bearing $(p<0.0001)$ and full weight-bearing $(p<0.0001)$ also demonstrated significant improvements in patients with LCP distal ulna hook plate fixation compared to IMS fixation. Patients in the LCP distal ulna hook plate fixation cohort had significantly increased AOFAS at 9 months $(p<0.0001)$ and 12 months $(p<0.0001)$ after surgery compared to the IMS fixation cohort.

Conclusion: In this retrospective cohort study, LCP distal ulna hook plate fixation as an alternative fixation method was better therapy for the displaced avulsion FMBFs compared to IMS fixation. LCP distal ulna hook plate fixation had a short surgery time and improved functional performance.
\end{abstract}

Keywords: Locking compression plate (LCP), Intramedullary screw (IMS), Fifth Metatarsal Base fractures (FMBFs)

\footnotetext{
* Correspondence: bone_ghost@hotmail.com

${ }^{\dagger}$ Equal contributors

Department of Orthopedic Surgery, Wuhan Orthopedic Hospital, Wuhan Puai Hospital, Huazhong University of Science and Technology, Hanzheng Street 473\#, Wuhan City, Hubei Province 430033, China
} 


\section{Background}

The base of fifth metatarsal was defined as the proximal $1.5 \mathrm{~cm}$ of the shaft distal to its articular surface. Fifth metatarsal base fractures (FMBFs) were the most common type of the fifth metatarsal fractures [1]. The fracture typically occurred when an adduction force was applied to the forefoot with the ankle plantarflexed. The base was usually divided anatomically into three zones [2] (its tuberosity, meta-diaphyseal junction and proximal shaft). Fractures in zone 1 were the most familiar ones which comprised about $93 \%$ of all the proximal fifth metatarsal fractures [1]. In our study, displaced avulsion FMBFs were defined as the displaced fractures in zone 1 [3, 4] (Fig. 1).

Most un-displaced avulsion FMBFs can be treated with conservative treatment $[3,5]$. Non-weight-bearing with cast immobilization has been the main treatment of this injury $[5,6]$. However, this injury often was a source of lost work productivity and associated with nonunion rates of $7 \%$ to $28 \%$ [2]. Fractures displaced more than $2 \mathrm{~mm}$ frequently required surgical treatment to achieve anatomic reduction of the articular surface, early weightbearing and restoration of peroneus longus and brevis tendons [2]. Surgery treatment of displaced avulsion FMBFs was challenging because of its subcutaneous location, comminuted fracture, its attachment of peroneus longus and brevis muscles and its weight-bearing function [3, 7]. Intramedullary screw (IMS) fixation was wildly used in FMBFs [7-9] (Fig. 2). Previous studies showed that the screw was larger in both diameter and length the better [10]. However, the comminuted or small displaced avulsion FMBFs were difficulty to treat with large IMS and function outcomes following IMS remained unsatisfactory because of late weightbearing [11]. Meanwhile, the IMS was associated with many complications, including lateral gapping, distraction of the fracture site and mal-reduction of the fractures [10, 12].

The Locking Compression Plate (LCP) distal ulna hook plate fixation was a novel alternative fixation method [13, 14] (Fig. 2). In addition, case series with LCP distal ulna hook plate fixation had shown improved clinical outcomes [14]. The purpose of this retrospective cohort study was to determine if LCP distal ulna hook plate fixation resulted in improved outcomes compared to the IMS fixation.

\section{Methods}

\section{Patient eligibility}

From July 2013 to July 2016, 42 patients (43 cases) with displaced avulsion Fifth Metatarsal Base Fractures were treated surgically and evaluated retrospectively. Ethical approval and informed consent from every single patient was obtained. Eligible patients were included in our study when they met the following criteria: (1) diagnosed with displaced (more than $2 \mathrm{~mm}$ ) avulsion FMBFs; (2) over 18 years of age and in full possession of their mental faculties; (3) 4 days or less after injury; and (4) be treated with LCP distal ulna hook plate fixation (Fig. 3) or IMS (Fig. 4) and be follow-up until 12 months.

Patients with the following condition were excluded: (1) soft tissue injury: open fracture type Gustilo-

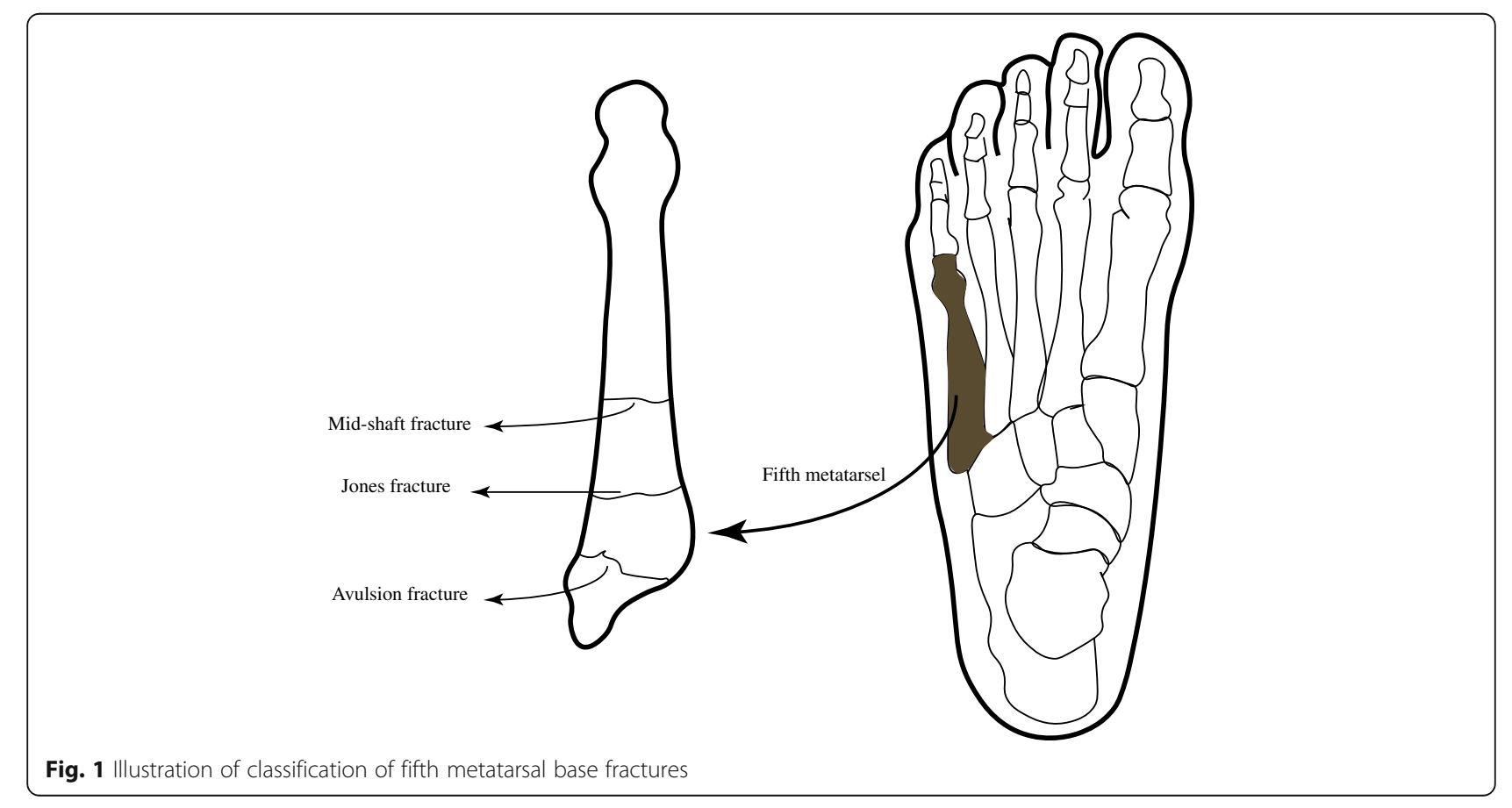




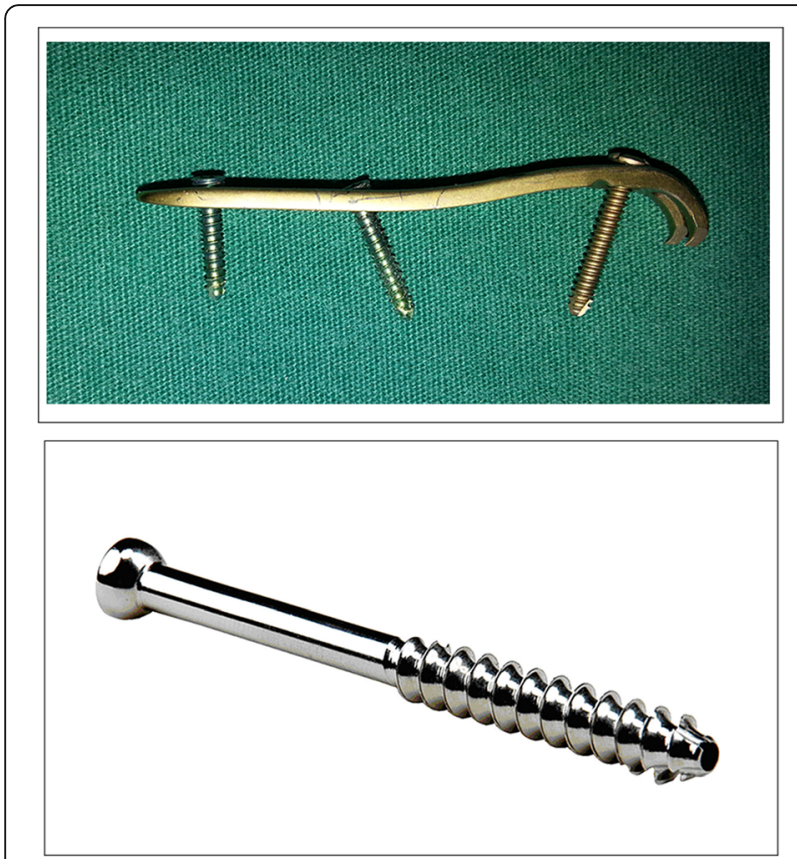

Fig. 2 Photograph of Locking Compression Plate (LCP) distal ulna hook plate and Intramedullary Screw

anderson Type II or higher; (2) pathological fracture or re-fracture; (3) additional zone 2 and 3 fractures; and (4) bilateral fracture.

\section{Surgical treatment and rehabilitation protocol}

All patients were surgically treated by a single, senior surgeon (ZHF) with the goals of anatomic reduction of the articular surface, achieve stable fixation and rebuilt the attachment of peroneus longus and brevis tendons. A lateral approach was used for direct visualization of the fractures as previously described. Patients in both cohorts were subject to the same postoperative rehabilitation protocol, which included no weight-bearing with short leg cast for 3 weeks. Radiographs were taken only on initial presentation to the clinic and in those patients with a great deal of pain clinically at the fracture site at the 6-week stage. Fracture union was defined radio graphically by bridging bone on at least 3 of 4 cortices.

\section{Clinical and function outcome assessments}

The baseline characteristics including age, male, and smoking were collected. Patient-reported weight-beating time, pain relief, and clinical functional outcomes were prospectively collected at predetermined intervals of $3,6,9$, and 12 months postoperatively. Subjective clinical outcomes were measured using the American Orthopedic Foot and Ankle Society Score (AOFAS). It was one of the most widely used clinician-reporting tools for foot and ankle conditions. Developed in 1994, AOFAS is

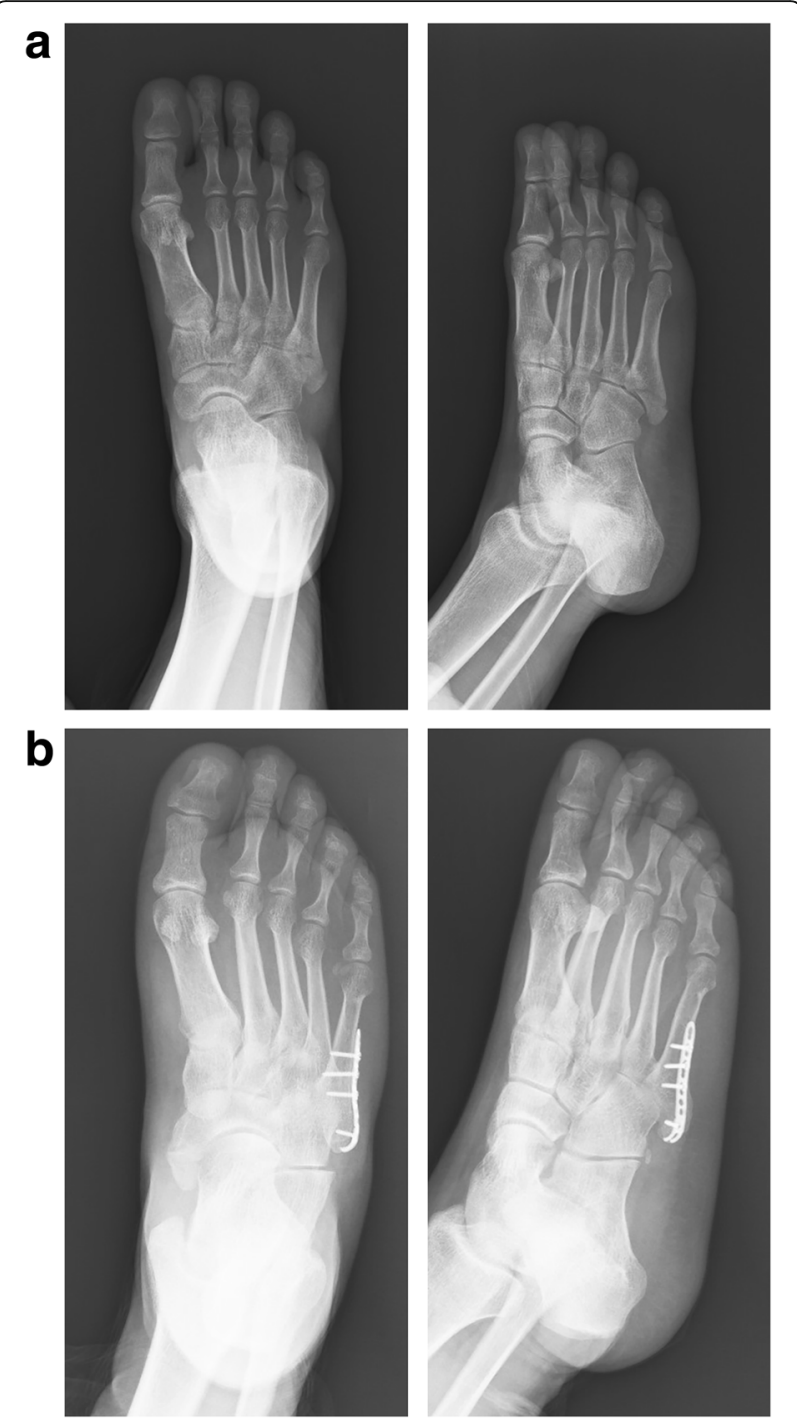

Fig. 3 Pre- (a) and postoperative (b) radiographs of a fifth metatarsal base fracture that was treated surgically using the Locking Compression Plate (LCP) distal ulna hook plate fixation

a clinician-based score that measures outcomes on four different anatomic regions of the foot: The anklehindfoot, midfoot, metatarsophalangeal (MTP)-interphalangeal (IP) for the hallux, and MTP-IP for the lesser toes. Complications were also collected in our studies until the 12 months' follow-up.

\section{Statistics}

Statistical analyses were performed using STATA, version 10.0 (Stata Corporation, College Station, Texas, USA). We summarized continuous data with means and standard deviations (SDs). The two groups were compared with regards to continuous and categorical outcomes using the non-parametric T-test and Chi-square 


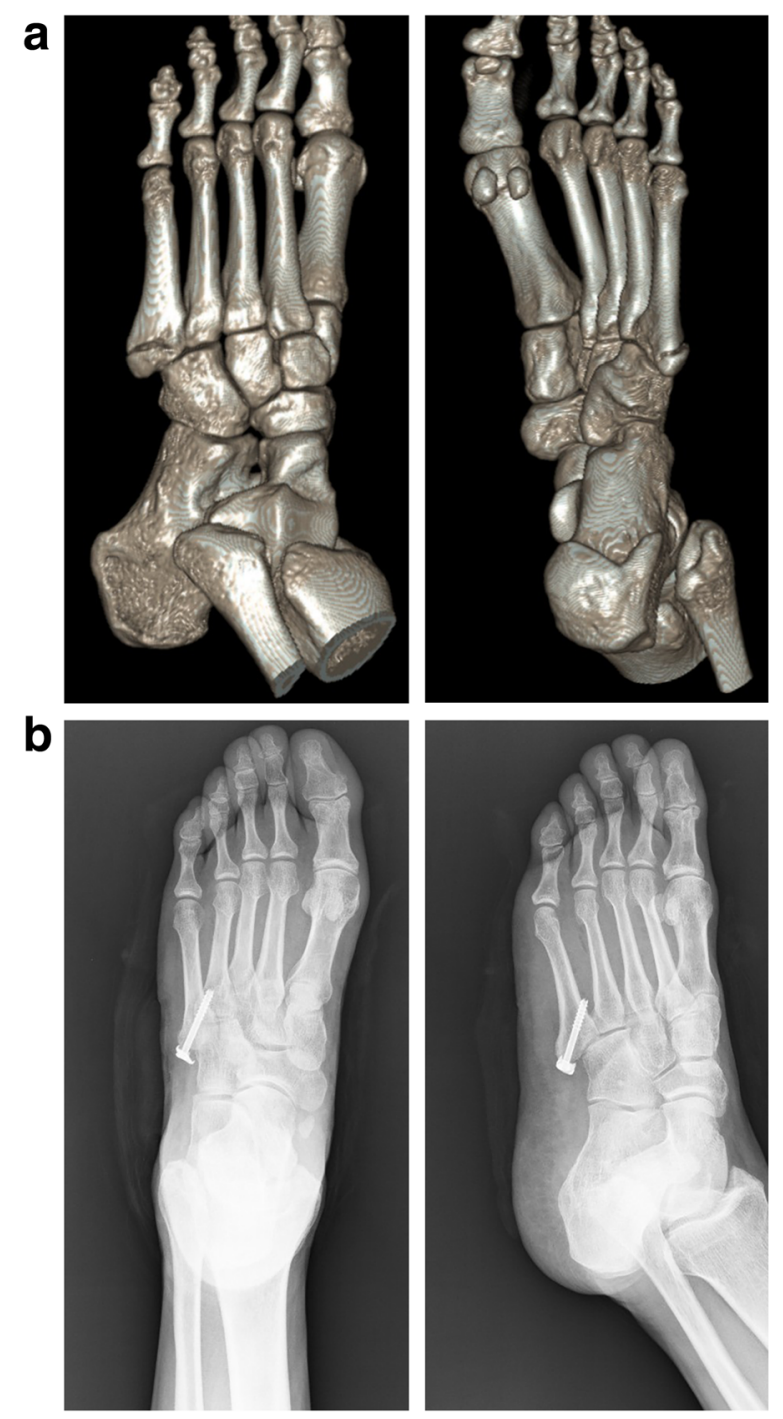

Fig. 4 Preoperative 3D reformations of CT scans (a) and postoperative (b) plain radiographs of a fifth metatarsal base fracture that was treated surgically using the Intramedullary Screw

test respectively. We considered the conventional level of statistical significance as $p<0.05$.

\section{Results}

18 patients treated with IMS and 25 patients treated with LCP distal ulna hook plate fixation were included in the study. All the raw data was in the Additional file 1. The two cohorts had similar baseline characteristics, including mean age (39.89 and 34.36 years, $p=0.4575$ ), gender distribution $(27.78 \%$ and $60.00 \%$ male, $p=0.5226)$, and smoking rates $(38.89 \%$ and $48.00 \%$, $p=0.5528$ ) (Table 1). For both cohorts, the surgery time was significantly less in patients with LCP distal ulna hook plate fixation cohort (40.94 and $53.5 \mathrm{~min}$, $p<0.001)$. Time for partial weight-bearing (3.67 and
Table 1 The baseline characters of patients

\begin{tabular}{lllll}
\hline & PF $n=18$ & IMS $n=25$ & $p$-Value & Test \\
\hline Age & $39.89 \pm 2.739$ & $34.36 \pm 1.977$ & 0.4575 & Unpaired T test \\
Male & $27.78 \%(5)$ & $60.00 \%(10)$ & 0.5226 & Chi-square test \\
Smoking & $38.89 \%(7)$ & $48.00 \%(12)$ & 0.5528 & Chi-square test
\end{tabular}

PF plate fixation, IMS Intramedullary screw

5.36 weeks, $\mathrm{p}<0.001)$, full weight-bearing (6.52 and 8.48 weeks, $\mathrm{p}<0.001)$, and bony union (7.49 and 9.64 weeks, $p=0.0053$ ) was significantly less in patients with LCP distal ulna hook plate fixation cohort compared to IMS cohort. There was no significant difference in the time for return to daily life (12 and 12.52 weeks, $p=0.4192)$, pain scores before surgery (7.72 and 8 , $p=0.2432)$, pain scores at 3 (4.61 and 4.64, $p=0.9072$ ), 6 (3.56 and 3.68, $p=0.4589), 9$ (2.39 and 2.68, $p=0.0856)$, and 12 weeks $(0.44$ and $0.52, p=0.6346)$ after surgery, AOFAS before surgery (43.61 and 44.24, $p=0.4554)$, AOFAS at 3 (74.17 and 74.16, $p=0.9931)$, 6 months (76.67 and 76.6, $p=0.7879$ ) after surgery between the two cohorts. While, the AOFAS scores at 9 (82.06 and 78.64, $p<0.0001$ ), 12 months (93.56 and $87.8, \mathrm{p}<0.0001)$ after surgery were significantly higher in patients with LCP distal ulna hook plate fixation cohort (Table 2). One patient immigrates to Canada, we missed the follow up. There was no significant difference in the complications after surgery between the two cohorts.

\section{Discussion}

The main finding of this study is the efficacity of the fixation using a LCP distal ulna hook plate for a novel approach. There were only 4 studies studied the plate in the treatment of FMBFs [13, 15-17]. Three studies had evaluated the results of surgical treatment of zones I and II FMBFs using a mini-hook plate $[13,15,16]$. The fourth investigated the biomechanical comparison of IMS versus Low-Profile Plate fixation of a jones fracture [17]. There has been no previous comparison study investigating pain and functional outcome following treatment with LCP distal ulna hook plate and IMS, and our study is the first comparative retrospective cohort study to compare LCP distal ulna hook plate and IMS in displace evulsion FMBFs. Our finding showed that LCP distal ulna hook plate fixation as an alternative fixation method was better therapy for the displaced avulsion FMBFs than IMS fixation. LCP distal ulna hook plate fixation had a short surgery time and improved functional performance.

FMBFs can be challenging because of its subcutaneous location [18]. IMS as a precuneus technical was widely used in clinic and accepted as the standard of surgical treatment of these fractures [12]. Meanwhile, the screw 
Table 2 Surgical Results of patients with Fifth Metatarsal Base Fracture

\begin{tabular}{|c|c|c|c|c|}
\hline & $\operatorname{PF}(n=18)$ & IMS $(n=25)$ & $P$ & Test \\
\hline Time for surgery* & $40.94 \pm 1.18$ & $53.52 \pm 0.66$ & $<0.0001$ & Unpaired T test \\
\hline Time to partial weight-bearing* & $3.67 \pm 0.14$ & $5.36 \pm 0.13$ & $<0.0001$ & Unpaired $T$ test \\
\hline Time to full weight-bearing* & $6.52 \pm 0.12$ & $8.48 \pm 0.19$ & $<0.0001$ & Unpaired $T$ test \\
\hline Time for bony union* & $7.49 \pm 0.10$ & $9.64 \pm 0.61$ & 0.0053 & Unpaired T test \\
\hline Time for return to daily life & $12 \pm 0.45$ & $12.52 \pm 0.42$ & 0.4192 & Unpaired $T$ test \\
\hline Pain before surgery & $7.72 \pm 0.21$ & $8 \pm 0.13$ & 0.2432 & Unpaired T test \\
\hline Pain 3 weeks & $4.61 \pm 0.18$ & $4.64 \pm 0.16$ & 0.9072 & Unpaired T test \\
\hline Pain 6 weeks & $3.56 \pm 0.15$ & $3.68 \pm 0.10$ & 0.4589 & Unpaired T test \\
\hline Pain 9 weeks & $2.39 \pm 0.14$ & $2.68 \pm 0.10$ & 0.0856 & Unpaired T test \\
\hline Pain 12 weeks & $0.44 \pm 0.12$ & $0.52 \pm 0.10$ & 0.6346 & Unpaired T test \\
\hline AOFAS before surgery & $43.61 \pm 0.56$ & $44.24 \pm 0.57$ & 0.4554 & Unpaired T test \\
\hline AOFAS 3 months & $74.17 \pm 0.63$ & $74.16 \pm 0.46$ & 0.9931 & Unpaired T test \\
\hline AOFAS 6 months & $76.67 \pm 0.19$ & $76.6 \pm 0.15$ & 0.7879 & Unpaired T test \\
\hline AOFAS 9 months* & $82.06 \pm 0.12$ & $78.64 \pm 0.11$ & $<0.0001$ & Unpaired T test \\
\hline AOFAS 12 months* & $93.56 \pm 0.25$ & $87.8 \pm 0.17$ & $<0.0001$ & Unpaired T test \\
\hline Complication delayed union & 2 & 3 & $>0.9999$ & Fisher's exact test \\
\hline Complication nonunion & 0 & 0 & - & - \\
\hline Complication infection & 0 & 0 & - & - \\
\hline
\end{tabular}

PF plate fixation, IMS Intramedullary screw, AOFAS American Orthopedic Foot and Ankle Society

*and bold means $P<0.05$

system was developed, allowing surgeons to choose among 4.5-, 5.5-, and 6.5-mm solid stainless steel screws. However, it was difficulty to fix the small avulsion fractures with screws and there were several complications associated with them, such as irritability of screws head, injury of peripheral nerve, bone nonunion because of small diameter, and secondary fractures because of large diameter [12]. Alternatively, LCP distal ulna hook plate may be a good choice. This plate has several advantages: (1) the fifth metatarsal tuberosity can be grasped tightly by the plate hook to maintain the stability of the peroneal tendons adhesion; (2) as a checkered plate, the re-displaced of fractures can be reduced. Joint surface collapse can be prevented by the support function of this plate; (3) this plate had good histocompatibility; (4) the fifth metatarsal's bending curvature fitted to the LCP distal ulna hook plate; and (5) low profile, obtuse edge and polishing surface can reduce the irritability of the soft tissue [17]. Vorlat, Achtergael and Haentjens reported that the most significant predictor of a poor functional outcome after these injuries was a prolonged period of non-weight-bearing [19]. The advantage functional outcome of LCP distal ulna hook plate was related to the early weight-bearing.

The retrospective cohort design of this study has several strengths. All cases were performed by a single senior surgeon (ZHF) using the same surgical approach, and all functional outcome evaluations were completed by a single senior physical therapist (SJZ) for precision. In addition, complications in both groups minimal. Limitations of this study include only twelve months of outcomes postoperatively, the total number of patients were small, and the radiographic evaluation was incomplete. Nowadays, we are focus on the effectively of the fifth metatarsal fractures anatomy plate.

\section{Conclusions}

This study suggests that the use of LCP distal ulna hook plate fixation improves patients' outcomes postoperatively.

\section{Additional file}

Additional file 1: The file contains the raw data of the age, gender, smoking, time for surgery, time for partial weight-bearing, time for full weight-bearing, time for bone union, return to daily life, pain before surgery, pain 3 weeks, pain 6 weeks, pain 6 weeks, pain 12 weeks, AOFAS before surgery, AOFAS 3 months, AOFAS 6 months, AOFAS 9 months, AOFAS 12 months, delayed union, nonunion, and infection. (XLSX $32 \mathrm{~kb}$ )

\section{Abbreviations}

AOFAS: American Orthopedic Foot and Ankle Society; FMBFs: Fifth Metatarsal Base Fractures; IMS: Intramedullary screw; LCP: Locking Compression Plate; PF: Plate Fixation

Acknowledgements

Not applicable. 


\section{Funding}

The author(s) received no financial support for the research, authorship, and/or publication of this article.

\section{Availability of data and materials}

All data generated or analyzed during this study are included in this published article.

\section{Authors' contributions}

ZHF and LX suggested the idea and were major contributors in writing the manuscript. XG and SJZ measured, analyzed and interpreted the patients' data. XG modified our manuscript. ZHF performed the surgery. All authors read and approved the final manuscript.

\section{Ethics approval and consent to participate}

The study was approved by the clinical research ethics committee of Wuhan Puai Hospital. Reference No. 2013-049-02.

\section{Consent for publication}

Not applicable.

\section{Competing interests}

The authors declare that they have no competing interests.

\section{Publisher's Note}

Springer Nature remains neutral with regard to jurisdictional claims in published maps and institutional affiliations.

Received: 30 June 2017 Accepted: 21 September 2017

Published online: 26 September 2017

\section{References}

1. Lawrence SJ, Botte MJ. Jones' fractures and related fractures of the proximal fifth metatarsal. Foot \& ankle. 1993;14(6):358-65.

2. Petrisor BA, Ekrol I, Court-Brown C. The epidemiology of metatarsal fractures. Foot Ankle Int. 2006:27(3):172-4.

3. Egol K, Walsh M, Rosenblatt K, Capla E, Koval KJ. Avulsion fractures of the fifth metatarsal base: a prospective outcome study. Foot Ankle Int. 2007;28(5):581-3.

4. Mehlhorn AT, Zwingmann J, Hirschmuller A, Sudkamp NP, Schmal H. Radiographic classification for fractures of the fifth metatarsal base. Skelet Radiol. 2014;43(4):467-74

5. Wiener BD, Linder JF, Giattini JF. Treatment of fractures of the fifth metatarsal: a prospective study. Foot Ankle Int. 1997;18(5):267-9.

6. Shahid MK, Punwar S, Boulind C, Bannister G. Aircast walking boot and below-knee walking cast for avulsion fractures of the base of the fifth metatarsal: a comparative cohort study. Foot Ankle Int. 2013:34(1):75-9.

7. Mahajan V, Chung HW, Suh JS. Fractures of the proximal fifth metatarsal: percutaneous bicortical fixation. Clinics in orthopedic surgery. 2011:3(2):140-6.

8. Mologne TS, Lundeen JM, Clapper MF, O'Brien TJ. Early screw fixation versus casting in the treatment of acute Jones fractures. Am J Sports Med. 2005;33(7):970-5.

9. Tan EW, Cata E, Schon LC. Use of a Percutaneous Pointed Reduction Clamp Before Screw Fixation to Prevent Gapping of a Fifth Metatarsal Base Fracture: A Technique Tip. J Foot Ankle Surg. 2016;55(1):151-6.

10. Wright RW, Fischer DA, Shively RA, Heidt RS Jr, Nuber GW. Refracture of proximal fifth metatarsal (Jones) fracture after intramedullary screw fixation in athletes. Am J Sports Med. 2000:28(5):732-6.

11. Roche AJ, Calder JD. Treatment and return to sport following a Jones fracture of the fifth metatarsal: a systematic review. Knee Surg Sports Traumatol Arthrosc. 2013;21(6):1307-15.

12. Ochenjele G, Ho B, Switaj PJ, Fuchs D, Goyal N, Kadakia AR. Radiographic study of the fifth metatarsal for optimal intramedullary screw fixation of Jones fracture. Foot Ankle Int. 2015;36(3):293-301.

13. Lee SK, Park JS, Choy WS. LCP distal ulna hook plate as alternative fixation for fifth metatarsal base fracture. Eur J Orthop Surg Traumatol. 2013;23(6):705-13.

14. Lorich DG, Fabricant PD, Sauro G, Lazaro LE, Thacher RR, Garner MR, Warner SJ. Superior Outcomes after Operative Fixation of Patella Fractures using a Nove Plating Technique: a Prospective Cohort Study. J Orthop Trauma. 2017;
15. Choi JH, Lee KT, Lee YK, Lee JY, Kim HR. Surgical results of zones I and II fifth metatarsal base fractures using hook plates. Orthopedics. 2013;36(1):e71-4.

16. Lee SK, Park JS, Choy WS. Locking compression plate distal ulna hook plate as alternative fixation for fifth metatarsal base fracture. J Foot Ankle Surg. 2014;53(5):522-8.

17. Huh J, Glisson RR, Matsumoto T, Easley ME. Biomechanical Comparison of Intramedullary Screw Versus Low-Profile Plate Fixation of a Jones Fracture. Foot Ankle Int. 2016:37(4):411-8.

18. O'Malley M, DeSandis B, Allen A, Levitsky M, O'Malley Q, Williams R. Operative Treatment of Fifth Metatarsal Jones Fractures (Zones II and III) in the NBA. Foot Ankle Int. 2016;37(5):488-500.

19. Vorlat P, Achtergael W, Haentjens P. Predictors of outcome of non-displaced fractures of the base of the fifth metatarsal. Int Orthop. 2007;31(1):5-10.

\section{Submit your next manuscript to BioMed Central and we will help you at every step:}

- We accept pre-submission inquiries

- Our selector tool helps you to find the most relevant journal

- We provide round the clock customer support

- Convenient online submission

- Thorough peer review

- Inclusion in PubMed and all major indexing services

- Maximum visibility for your research

Submit your manuscript at www.biomedcentral.com/submit
Biomed Central 